\title{
Fatigue design of cast iron components with surface discontinuities
}

\author{
Márton Gróza ${ }^{1, *}$, Yves Nadot $^{2}$, and Károly Váradi ${ }^{1}$ \\ ${ }^{1}$ TU Budapest, Department of Machine and Product Design, Müegyetem rkp. 3, Budapest 1111, Hungary \\ ${ }^{2}$ Institut Pprime, CNRS, ISAE-ENSMA, Université de Poitiers, Téléport 2, 1 Avenue Clément Ader, 86961 Futuroscope \\ Chasseneuil Cedex, France
}

\begin{abstract}
Surface discontinuities are unavoidable properties of production castings, and have fundamental effect on in-service reliability. In the current study a multiaxial probabilistic fatigue analysis model has been developed for the description of local (e.g.: notches or surface defects) and structural gradient (e.g: bending) effects on crack initiation life. Identification of the model parameters and the validation has been done on the ferrite-pearlitic NCI grade EN GJS 500-7. The survival probability type simulation results are proven to be highly accurate with $5 \%$ average, and $11 \%$ maximum prediction error. In theory, the model might be able to assess the effect of arbitrarily complex defects on the fatigue strength, which is to be investigated in the future.
\end{abstract}

\section{Introduction}

Nodular cast irons (NCI) are widely used due to their favorable mechanical properties coupled with economical manufacturability of components with complex geometrical features. One of the most important design goals of NCI components in the transportation industry is to ensure safe in-service life. Fatigue is strongly linked with surface quality, which is a wellknown weakness of NCI. Shrinkages, surface pores and gas blisters are frequent on cast surfaces, and they are usually responsible for the initiation of the fatigue process.

From a mechanical standpoint the different types of surface defects are usually modelled as notches or equivalent cracks. A large number of deterministic approaches based on the continuum and fracture mechanics have been developed over the years, from which probably the semi-empirical approach from Murakami [1] is the best known in the scientific and industrial community.

Due to the large number of parameters involved (material properties and component geometry, load history, environmental effects) result scatter is inseparable from the cyclic loading governed material degradation process.

One way to describe the experienced scatter in the fatigue strength and life is to account for the uncertainties of the input parameters, and use the established deterministic models for the computation of the expected value and the deviation. Beretta and Regazzi [2] developed a probabilistic extension to the standard railway axle calculation method which computes failure probability utilizing Monte Carlo simulations considering the position of the $\mathrm{S}-\mathrm{N}$ curve and the load spectrum as random variables. Kim et al. [3] analyzed multiple crack growth with the NASGRO model and XFEM simulations considering the variability of initial crack length, material parameters and crack growth rates.

Probabilistic fatigue models are usually built with the introduction of different damage parameters into specific distribution functions. Pessard et al. [4] have built a probabilistic modelling framework for the description of the Kitagawa diagram where different damage parameters are employed for crack initiation and threshold crack propagation. The weakest link hypothesis is then applied to compute the probability of failure from the two damage mechanisms. The two parameter Weibull distribution is used to describe the threshold of the damage parameter corresponding to the failure of one grain.

Correia et al. [5] introduced several damage parameters into a general probabilistic model using the three parameter Weibull distribution. Their model with energy and critical plane based fatigue damage parameters led to promising results for multiaxial loading conditions as well.

In the current paper the probabilistic approach introduced in Simon's PhD Thesis [6] for the modelling of the notch effect under proportional high-cycle fatigue conditions is developed further. The updated formulation leads to more accurate description of the notch effect on the high-cycle fatigue strength considering both the expected value and the scatter of results. The subject of the study is a ferritic-pearlitic nodular cast iron, which classifies as grade EN GJS 500-7 according to EN $1563: 2012$ [7].

\footnotetext{
Corresponding author: groza.marton@gt3.bme.hu
} 


\section{Experimental results}

To identify the parameters of the probabilistic model introduced in Section 3. experimental data is needed related to stress multiaxiality and the notch effect, and information related to the scatter of the fatigue strength.

The experimental results concerning the notch, load ratio and load type effects are derived from the work of Deguchi et al. [8]-[10]. Since this data cannot be used to draw conclusions related to result scatter, a set of experimental data is introduced by the authors for the same material grade to analyze of the estimated result scatter.

\subsection{Notch, load ratio and load type effects}

Deguchi et al. [8]-[10] introduced a set of experimental data related to a ferrite-pearlitic nodular cast iron material with $10.5 \%$ graphite, $45.3 \%$ ferrite, and $44.2 \%$ perlite content. The investigated material classifies as grade EN GJS 500-7 according to EN 1563:2012 [7] with a yield strength of $R_{p 0.2}=335[\mathrm{MPa}]$, an ultimate tensile strength of $R_{m}=552$ [MPa].

Rotating bending and tension compression fatigue tests were conducted on circumferentially notched and unnotched polished specimens, and on specimens with a drilled hole. Fig. 1 shows the geometry of the test specimens. The fatigue limit was determined as the stress amplitude which the specimen has endured for $10^{7}$ load cycles. Table 1. summarizes the test results, where $\mathrm{D}$ is the diameter of the proof section, $\sigma_{a}^{10^{7}}$ is the stress amplitude leading to the survival of $10^{7}$ load cycles (fatigue limit), RB designates rotating-bending, $\mathrm{T}$ stands for pulsating tension, $\mathrm{TC}$ is for tension-compression, $\mathrm{R}$ is the load ratio, $t$ is the notch depth, $D$ is the diameter of the drilled hole and $h$ is the depth of the drilled hole.

\subsection{Scatter of fatigue strength}

Fatigue tests were conducted at three different load ratios (R-1, R0.05 and R0.5) on cylindrical polished EN GJS 500-7 ferrite-pearlitic nodular cast iron test specimens for the investigation of the mean stress effect. The investigated material contained $12.8 \%$ graphite, $51.5 \%$ ferrite, $35.7 \%$ pearlite, and the basic mechanical properties were: $R_{p 0.2}=325[\mathrm{MPa}], R_{m}=504[\mathrm{MPa}]$ and the elongation at fracture $A=7.1[\%]$. The chemical composition is detailed in Table 2., whereas Fig. 2 shows the microstructure of the material on LOM and SEM images. Fatigue tests were conducted at $22^{\circ} \mathrm{C}$ room temperature with a force controlled $30 \mathrm{~Hz}$ sinusoidal load curve on an Instron 8874 servohydraulic fatigue testing machine according to ISO 1099:2017 [11]. The fatigue limit $\left(\sigma_{a}^{10^{6}}\right)$ has been determined with the stepby-step method [12] for $10^{6}$ load cycles. The fracture surfaces were analyzed by means of optical and electron microscopy. The crack initiation sites were small subsurface defects not exceeding the $100 \mu m$ size measured in the $\sqrt{\text { area }}$ parameter from Murakami [1]. Specimen geometry is shown in Fig. 3, results of the fatigue tests are summarized in Table 3.

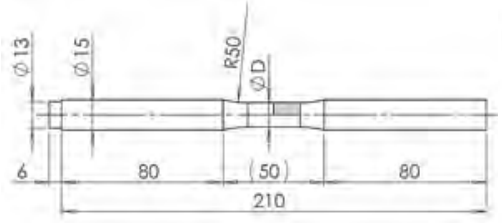

a)

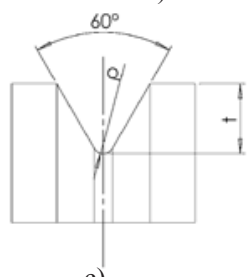

c)

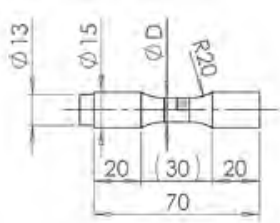

b)
Fig. 1. Geometry of test specimens [8]-[10], a.) rotating bending specimen, b.) tension compression specimen, c.) circumferential notch geometry, d.) drilled hole geometry.

Table 1. Summary of test results [8]-[10].

\begin{tabular}{|c|c|c|c|}
\hline Load type & $\mathrm{D}[\mathrm{mm}]$ & Specimen type & $\sigma_{a}^{10^{7}}[\mathrm{MPa}]$ \\
\hline $\mathrm{RB}, \mathrm{R}=-1$ & 10 & smooth & 260 \\
\hline $\mathrm{T}, \mathrm{R}=0.1$ & 10 & smooth & 170 \\
\hline \multirow{2}{*}{$\mathrm{RB}, \mathrm{R}=-1$} & \multirow{2}{*}{12} & notched, $\mathrm{t}=100 \mu \mathrm{m}$ & 195 \\
\cline { 3 - 4 } & & notched, $\mathrm{t}=200 \mu \mathrm{m}$ & 165 \\
\cline { 3 - 4 } & & notched, $\mathrm{t}=1000 \mu \mathrm{m}$ & 92.5 \\
\hline $\mathrm{TC}, \mathrm{R}=-1$ & 15 & notched, $=2500 \mu \mathrm{m}$ & 52.5 \\
\hline \multirow{2}{*}{$\mathrm{T}, \mathrm{R}=0.1$} & \multirow{2}{*}{15} & notched, $\mathrm{t}=100 \mu \mathrm{m}$ & 125 \\
\cline { 3 - 4 } & & notched, $=2500 \mu \mathrm{m}$ & 45 \\
\hline \multirow{2}{*}{$\mathrm{RB}, \mathrm{R}=-1$} & \multirow{2}{*}{12} & hole, $\mathrm{d}=\mathrm{h}=200 \mu \mathrm{m}$ & 220 \\
\cline { 3 - 4 } & & hole, $\mathrm{d}=\mathrm{h}=500 \mu \mathrm{m}$ & 175 \\
\hline \multirow{2}{*}{$\mathrm{T}, \mathrm{R}=0.1$} & \multirow{2}{*}{15} & hole, $\mathrm{d}=\mathrm{h}=200 \mu \mathrm{m}$ & 150 \\
\cline { 3 - 4 } & & hole, $\mathrm{d}=\mathrm{h}=500 \mu \mathrm{m}$ & 120 \\
\hline
\end{tabular}

Table 2. Average chemical composition of the test specimens.

\begin{tabular}{|c|c|c|c|c|c|c|c|c|}
\hline $\mathrm{C}$ & $\mathrm{Si}$ & $\mathrm{Mn}$ & $\mathrm{P}$ & $\mathrm{S}$ & $\mathrm{Cr}$ & $\mathrm{Cu}$ & $\mathrm{Mg}$ & $\mathrm{Ni}$ \\
\hline 3.57 & 2.1 & 0.18 & 0.023 & 0.006 & 0.02 & 0.32 & 0.031 & 0.03 \\
\hline
\end{tabular}
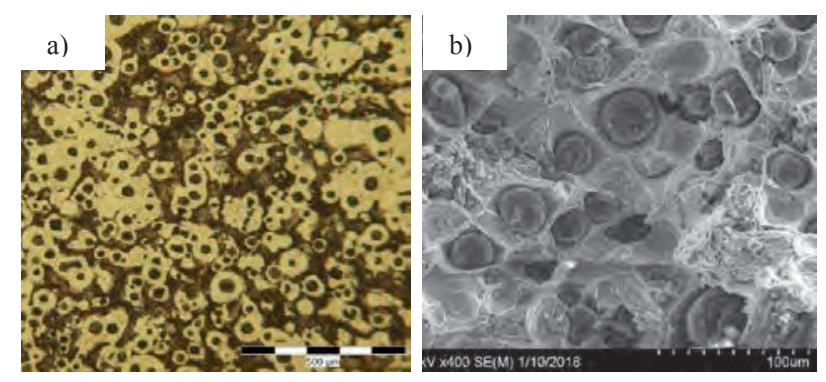

Fig. 2. a) LOM cross-sectional micrograph 50x, b) SEM image from the fracture surface of a fatigue test specimen $400 \mathrm{x}$.

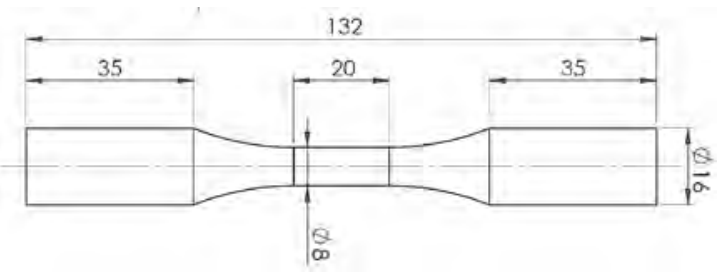

Fig. 3. Geometry of cylindrical polished test specimens. 
Table 3. Summary of test results, estimated stress amplitudes at $10^{6}$ load cycles and the corresponding Crossland equivalent stress.

\begin{tabular}{|c|c|c|c|c|c|}
\hline \multicolumn{2}{|c|}{$\mathrm{R}=-1$} & \multicolumn{2}{c|}{$\mathrm{R}=0.05$} & \multicolumn{2}{c|}{$\mathrm{R}=0.5$} \\
\hline $\begin{array}{c}\sigma_{a}^{10^{6}} \\
{[\mathrm{MPa}]}\end{array}$ & $\begin{array}{c}\sigma_{C r}^{10^{6}} \\
{[\mathrm{MPa}]}\end{array}$ & $\begin{array}{c}\sigma_{a}^{10^{6}} \\
{[\mathrm{MPa}]}\end{array}$ & $\begin{array}{c}\sigma_{C r}^{10^{6}} \\
{[\mathrm{MPa}]}\end{array}$ & $\begin{array}{c}\sigma_{a}^{10^{6}} \\
{[\mathrm{MPa}]}\end{array}$ & $\begin{array}{c}\sigma_{C r}^{10^{6}} \\
{[\mathrm{MPa}]}\end{array}$ \\
\hline 258 & 308 & 146 & 274 & 99 & 301 \\
\hline 252 & 300 & 152 & 284 & 97 & 294 \\
\hline 244 & 291 & 154 & 288 & 99 & 300 \\
\hline 247 & 295 & 151 & 273 & 98 & 297 \\
\hline 263 & 314 & - & - & 99 & 301 \\
\hline
\end{tabular}

In order to obtain comparable data from the tests conducted at three different load ratios, the corresponding Crossland equivalent stress $\left(\sigma_{C r}^{10^{6}}\right)$ has been calculated for each data point. The choice of the Crossland equivalent definition is justified by the nature of the analysis; the aim is to analyze high cycle fatigue behavior under proportional loading conditions. The Crossland equivalent stress $\left(\sigma_{C r}\right)$ is defined:

$$
\sigma_{C r}=\sqrt{J_{2, a}}+\alpha_{C r} \sigma_{h, \max },
$$

where $\sqrt{J_{2, a}}$ is the square root of the amplitude of the second invariant of the deviatoric stress tensor, $\sigma_{h, \max }$ is the maximum of the hydrostatic stress in each load cycle and $\alpha_{C r}$ is a model parameter. The identification process is illustrated in Fig. 4, and the value of $\alpha_{C r}$ is 1.85 .

In the probabilistic model introduced in Section 3, the two parameter Weibull distribution is utilized to describe the scatter of the fatigue limit. The probability density function of the Weibull distribution is given by:

$$
f(x ; \lambda, k)=\left\{\begin{array}{ll}
1-e^{-(x / \lambda)^{k}} & x \geq 0 \\
0 & x \leq 0
\end{array},\right.
$$

where $x$ is the variable ( $\sigma_{C r}^{10^{6}}$ in the current case), $\lambda$ is the scale and $k$ is the shape parameter. The maximum likelihood estimators describing both result scatter and inaccuracy of the Crossland criterion are $\lambda=299.54 \mathrm{MPa}$ and $k=30.49$. The dispersion of the corresponding Crossland equivalent stress corresponding to the fatigue limit $\sigma_{C r}^{10^{6}}$ is displayed in Fig. 5 alongside the fitted Weibull probability distribution function.

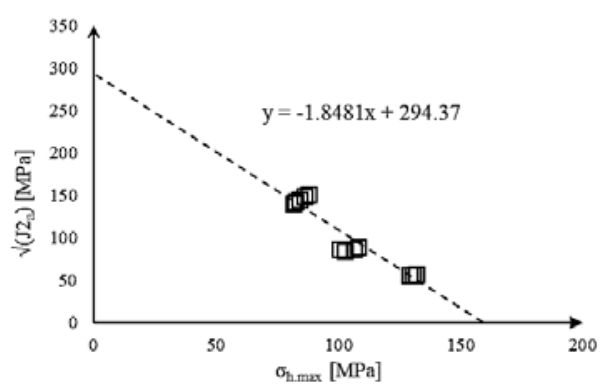

Fig. 4. Identification of the Crossland parameters on the $\sigma_{h, \max }-\sqrt{J_{2, a}}$ plane based in $\mathrm{R}=-1, \mathrm{R}=0.05$ and $\mathrm{R} 0.5$ tensile fatigue test results at $10^{6}$ cycles.

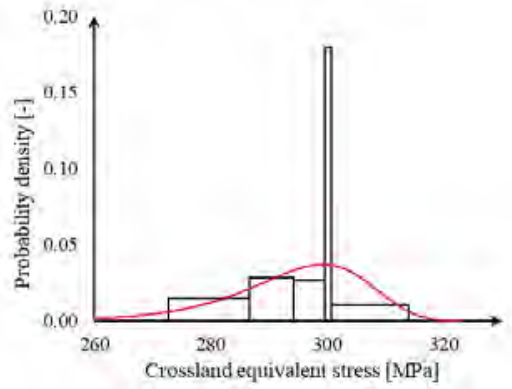

Fig. 5 Histogram and fitted Weibull distribution for the fatigue strength at $10^{6}$ cycles.

\section{Probabilistic approach}

The first iteration of the current probabilistic model was introduced in by Simon [6]. The current probabilistic criterion is based on the spatial distribution of the damage parameter and a selected distribution function. Due to the current application (proportional high cycle fatigue loading) the chosen damage parameter is the Crossland criterion. The choice of the two parameter Weibull distribution is justified by its convenient application with the weakest link theory and the low amount of parameters to identify.

In general, the probability of survival according to the two parameter Weibull distribution function:

$$
S(x ; \lambda, k)= \begin{cases}e^{-(x / \lambda)^{k}} & x \geq 0 \\ 1 & x \leq 0\end{cases}
$$

where $x$ is the variable, $\lambda$ and $k$ are the scale the shape parameters, respectively. Let this function define the probability of survival for one infinitesimal volume element in the component.

According to the weakest link theory, the failure of the weakest link leads to the failure of the whole structure. Similarly, the survival probability of the component is the product of the survival probability of the infinitesimal volume elements:

$$
S_{\text {comp }}=\prod_{\text {comp }} S(x ; \lambda, k) .
$$

The following substitutions introduce the mechanical variables into the distribution function: $x:=\sigma_{e q}, \lambda:=$ $\sigma_{u} \sqrt[m]{V_{\text {ref }} / \ln 2}$ and $k=m$. The equivalent stress $\sigma_{e q}$ is the Crossland equivalent stress according to Equation 1. The $\sigma_{u}$ is the ultimate value of $\sigma_{e q}$, which is denoted as $\beta_{C r}$ in the Crossland criterion. The reference volume $V_{\text {ref }}$ is linked with $\sigma_{u}$, the ultimate equivalent stress leads to failure in the reference volume. In this context the failure event is crack initiation.

The survival probability of the $i^{\text {th }}$ infinitesimal volume element:

$$
S_{E_{i}}=\left\{\begin{array}{cc}
1 & \sigma_{e q}<0 \\
e^{-\ln 2\left(\frac{\sigma_{e q}\left(E_{i}\right)}{\sigma_{u}}\right)^{m} \frac{1}{V_{r e f}}} & 0 \leq \sigma_{e q} \leq \sigma_{c} . \\
e^{-\ln 2\left(\frac{\sigma_{c}}{\sigma_{u}}\right)^{m} \frac{1}{V_{r e f}}} & \sigma_{c}<\sigma_{e q}
\end{array}\right.
$$

For equivalent stresses below $0 \mathrm{MPa}$, the survival probability of the volume element is 1 . Between 0 and the critical value $\sigma_{c}$ the survival probability is computed 
according to the distribution function. The critical equivalent stress $\sigma_{c}$ is a model parameter, which has been introduced to achieve more accurate results, which are less effected from localized disturbances in the stress field. Equivalent stresses above $\sigma_{c}$ lead to the same survival probability for an element of a fixed size.

The survival probability of the component is the product of the elemental survival probabilities:

$$
S_{\text {comp }}=\prod_{E_{i}} S_{E_{i}} \text {. }
$$

In the current evaluation the volume discretization into infinitesimal volume elements will be linked with the FE (Finite Element) mesh used for stress computation.

\subsection{Parameter identification}

The probabilistic approach requires parameter identification for the damage parameter, and for the distribution function.

\subsubsection{Identification of the Crossland parameters}

The parameters of the Crossland criterion can be determined with a least squares linear fit on the test data displayed on a $\sqrt{J_{2, a}}-\sigma_{h, \max }$ plot. The parameters have been identified on the unnotched results at the load ratios R-1 and R0.1 listed in Table 1. The identified parameters are $\alpha_{C r}=1.32$ and $\beta_{C r}=265 \mathrm{MPa}$.

\subsubsection{Identification of $\sigma_{u}$ and $V_{\text {ref }}$}

The ultimate value of the equivalent stress is $\sigma_{u}=\beta_{C r}$, which is the consequence of the applied Crossland criterion. The reference volume is the quarter of the proof volume of the unnotched specimens, in order to be coherent with the FE models built for the stress computation in the notched specimens: $V_{\text {ref }}=504 \mathrm{~mm}^{3}$.

\subsubsection{Identification of the $m$ parameter}

In the first formulation from Simon [6] the $m$ parameter was used in the identification process on notched fatigue results. This approach led to a good description of the notch effect, but the ability to describe result scatter was impaired. In the current formulation $m$ is identified on distribution of the fatigue limit expressed in the used equivalent stress parameter. Through the identification of $m$ on a different set of experimental data, the aim was to gain a realistic description of result scatter in the model. With the process detailed in Section $2.2 m=k=30.49$.

\subsubsection{Identification of the $\sigma_{c}$ parameter}

The critical equivalent stress $\sigma_{c}$ was introduced as parameter in order to readjust the model, since $m$ is not used as a fitting parameter for the accurate description of the notch effect any more. Using a limiting value for the linear stresses has the beneficial effect of leading to more robust results, which are less dependent on localized stress peaks and mesh quality. The identification has been done through minimization of errors, the value of $\sigma_{c}$ is $420 \mathrm{MPa}$ in the current application. Using one notched result as identification point would lead to different model prediction results.

\section{Calculations}

Linear elastic FE simulation models were built in ANSYS Mechanical to compute elemental stress results for each specimen and load scenario, which are necessary for the probabilistic model. The post processing of results to obtain elemental survival probabilities has also been coded in ANSYS.

The symmetry of the geometry and load conditions made it possible to utilize quarter symmetry of the mechanical problem. Analysis setup is displayed in Fig. 6, the modelled portion of the test specimen is shown as a dashed area in Fig. 1. Tension compression load scenarios are modelled with a distributed load at the end surface of the proof section, rotating bending is simulated with a moment load. In accordance with the method from Deguchi et al. [8]-[10], loads are considered to lead to the reported stress amplitudes on a cross-section corresponding the nominal proof section diameter for the specimens with drilled holes and small notches. For larger notch depths $(t=1000$ and $2500 \mu m)$ the actual cross section is considered when computing tensile and bending stresses.

At the bottom of the drilled holes a fictitious rounding of $50 \mu \mathrm{m}$ has been introduced, in order to obtain a more realistic representation of the drilled hole geometry and a non-singular stress field.

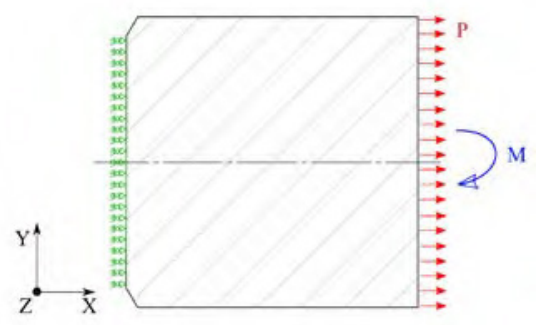

Fig. 6. Analysis setup for the FE computations.

Mesh convergence studies have been conducted for the circumferential notches and the drilled holes. Considering ensured mesh convergence and solution stability notched specimens were meshed with 20 node hexahedron elements (SOLID186), with mesh size defined by a 7.5 deg angle between nodes (midside nodes considered) along the $50 \mu \mathrm{m}$ radius at the bottom of the notches of various depth. Specimens with drilled holes were meshed with 10 node tetrahedrons (SOLID 187) with a mesh size of $R / 25 \mu \mathrm{m}$. The ferrite-pearlitic NCI material is considered with the modulus of elasticity $E=169 \mathrm{GPa}$ and Poisson's ratio $v=0.275$ material parameters in the calculation models. For each analysis model a half load cycle is simulated for the computation of the stresses corresponding to the load maximum and amplitude.

Fig. 7.a shows the mean elemental Crossland equivalent stress results (calculated according to Equation 
1.) for the rotating bending test specimen with $1000 \mu \mathrm{m}$ notch depth. Fig. 7.b) introduces the cut-off corresponding to the $\sigma_{c}$ critical equivalent stress model parameter. Fig. 8 shows the same results for the rotating bending specimen. Fig. 9 shows the computed elemental survival probability (calculated according to Equation 5.) for the rotating bending specimen with a notch depth of $1000 \mu \mathrm{m}$.
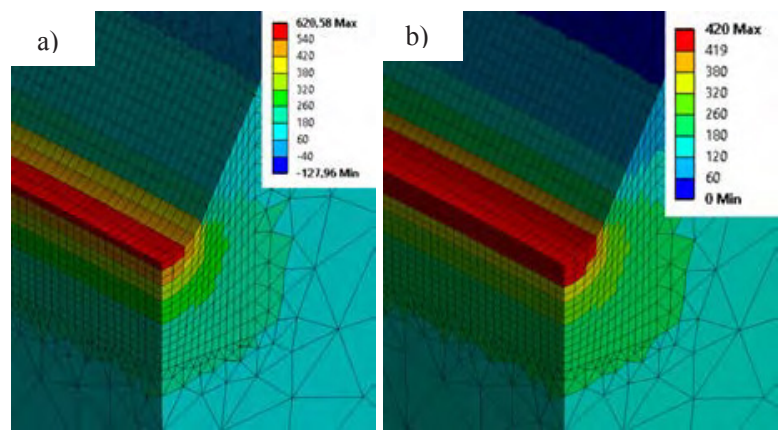

Fig. 7.a) Mean elemental Crossland equivalent stress results, b) Mean elemental Crossland equivalent stress results with the lower $(0)$ and upper $\left(\sigma_{c}\right)$ cut-off for the $\mathrm{RB} t=1000 \mu \mathrm{m}$ specimens.
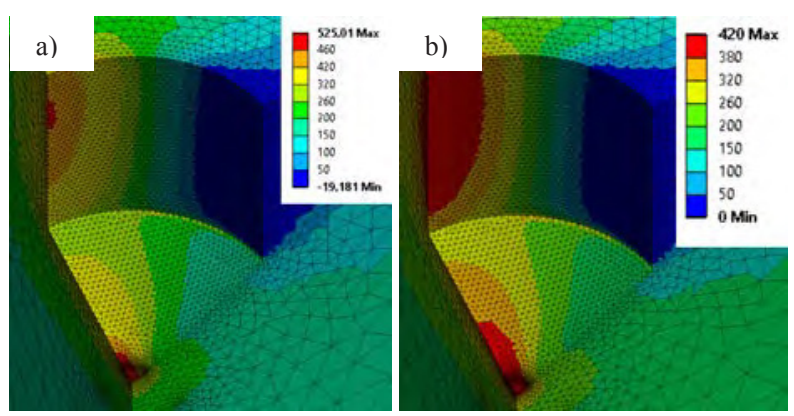

Fig. 8.a.) Mean elemental Crossland equivalent stress results, b.) Mean elemental Crossland equivalent stress results with the lower $(0)$ and upper $\left(\sigma_{c}\right)$ cut-off for the $\mathrm{RB} \mathrm{d}=\mathrm{h}=500 \mu \mathrm{m}$ specimens.

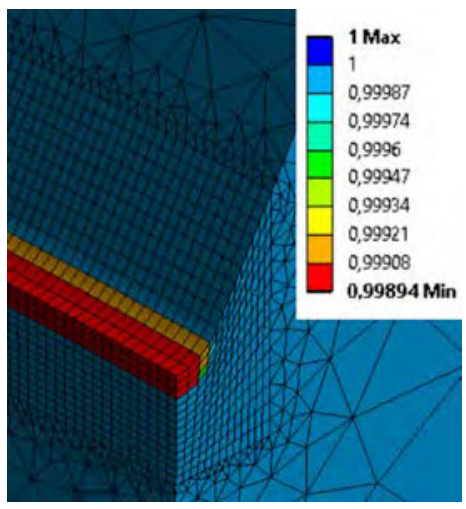

Fig. 9. Elemental survival probability for the $R B t=1000 \mu m$ specimen.

\section{Results and discussion}

Comparison of computational and experimental results is shown in Fig. 10. Each simulation leads to cumulative distribution function type survival probability curves as function of the applied stress amplitude during testing. The curves corresponding to the unnotched specimens are in perfect fit with the experiments since they have been used in the identification process. The computational results are in excellent agreement with the experiments for different load types (rotating bending, alternating tension-compression and pulsating tension), notch types (circumferential and drilled hole) and sizes $(100-2500 \mu \mathrm{m})$. Fig. 11. illustrates the prediction accuracy of the model, the average and maximum prediction error is 5 and $11 \%$, respectively.

In addition to the accurate description of local and structural gradient effects, the scatter of the fatigue limit is predicted in a correct manner. For a quantitative evaluation of the prediction accuracy related to result scatter a large experimental data set would be required with more than 10 specimens tested for every test case.

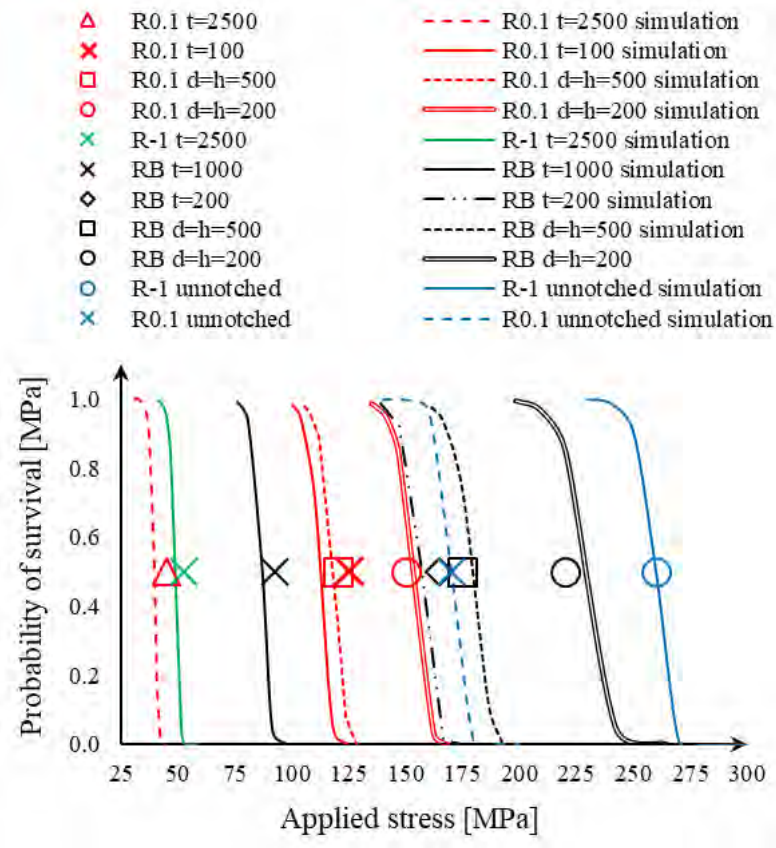

Fig. 10. Comparison of experimental and simulated survival probabilities.

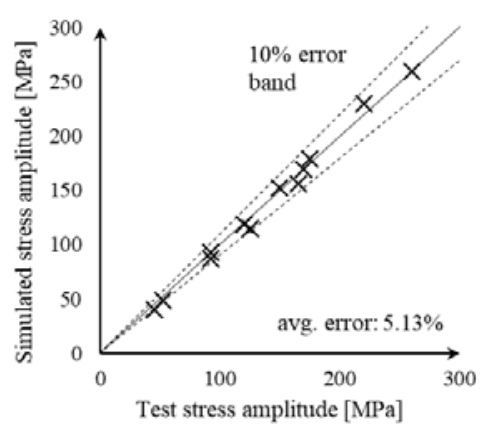

Fig. 11. Model prediction accuracy. 


\section{Conclusion}

A multiaxial probabilistic fatigue analysis model has been developed for the description of local and structural gradient effects on crack initiation. Identification of the model parameters and the validation has been done on the ferrite-pearlitic NCI grade EN GJS 500-7 [7].

- The probabilistic model describes load ratio, structural and local stress gradient effects induced by notches and surface holes of different size without the need for elastoplastic computation. The average and maximum estimated prediction error was 5 and $11 \%$, respectively.

- The model gives not only deterministic answers; it predicts the result scatter to be expected for a certain test case. The results scatter description accuracy has not been quantitatively evaluated.

- Theoretically, the model is able to describe the effect of arbitrarily complex defects and notches, and is not highly dependent on mesh quality. Further analyses are to be conducted to investigate these possibilities.

- Due to the application of the Crossland criterion as damage parameter, the application is limited to high cycle fatigue and proportional loading conditions in the current formulation.

This recent study was realized within the Knorr-Bremse Scholarship Program supported by the Knorr-Bremse Rail Systems Budapest.

\section{References}

[1] Y. Murakami, Metal Fatigue: Effects of Small Defects and Nonmetallic Inclusions, Oxford: Elsevier Science Ltd, 2002.

[2] S. Beretta and D. Regazzi, Int. J. Fatigue, 86, pp. 13-23, (2016)

[3] J. H. Kim, T. Chau-Dinh, G. Zi, W. W. Lee, and J. S. Kong, Eng. Fract. Mech., 156, pp. 182-196, (2016)

[4] E. Pessard, D. Bellett, F. Morel, and I. Koutiri, Eng. Fract. Mech., 109, pp. 89104, (2013)

[5] J. Correia, N. Apetre, A. Arcari, A. De Jesus, M. Muñiz-Calvente, R. Calçada, F. Berto, and A. Fernández-Canteli, Int. J. Fatigue, 100, pp. 187-194, (2017)

[6] S. Julien, PhD Thesis: Influence de microentailles sur le comportement en fatigue à grand nombre de cycles d'un alliage de TA6V; comparaison avec le frettingfatigue ISAE-ENSMA, (2017)

[7] EN 1563:2012 Founding - Spheroidal graphite cast irons. (2012)

[8] T. Deguchi, Adv. Exp. Mech., 2, pp. 8791, (2017)

[9] T. Deguchi, H. J. Kim, T. Ikeda, and K. Yanase, Influence of mean stress on fatigue strength of ferritic-pearlite ductile cast iron with small defects, J. Phys. Conf. Ser., 843, no. 1, (2017)

[10] T. Deguchi, H. J. Kim, and T. Ikeda, Fatigue limit prediction of ferriticpearlitic ductile cast iron considering stress ratio and notch size, J. Phys. Conf. Ser., 842, no. 1, 2017.

[11] ISO 1099:2017 Metallic materials Fatigue testing - Axial force-controlled method. (2017)

[12] R. S. Bellows, S. Muju, and T. Nicholas, Int. J. Fatigue, 21, no. 8, pp. 687-697, (1999) 